\section{The yin and the yang of immunosuppression with inhaled corticosteroids}

\author{
Ian Sabroe, ${ }^{1,2}$ Dirkje Postma, ${ }^{3,4}$ Irene Heijink, ${ }^{3,4,5}$ David H Dockrell ${ }^{2,6}$
}

A patient who is immunosuppressed is susceptible to mycobacterial infection: are you surprised? Before you jump to conclusions, you might wish to clarify the nature and extent of immunosuppression, and whether it is through disease, therapy, or both, reserving judgement until you know more.

Patients are often concerned about immunosuppressive effects of inhaled and oral corticosteroids. Asthmatics are used to infections putting them in hospital, and not unreasonably view with suspicion any treatment option that might make this more likely to happen. However, healthcare providers know that for asthmatics, appropriate local immunomodulation in the lung with inhaled corticosteroids (ICS) is associated with decreased exacerbations arising from viral infections, fewer symptoms, improved lung function and better outcomes. In subsets of patients with COPD, high-dose fluticasone with salmeterol reduces exacerbations and improves quality of life. ${ }^{1}$ Thus, if the only exposure of patients to anything remotely immunosuppressive is ICS, you might conclude the extent of immunosuppression is insufficient to regard the patient as immunocompromised.

But will use of ICS increase susceptibility to some infections? The innate immune networks active in the lung, with their multifaceted humoral and cellular components, show intrinsic competence in keeping the human organism mostly free of serious pulmonary infection. At

\footnotetext{
${ }^{1}$ Academic Unit of Respiratory Medicine, University of Sheffield, Sheffield, UK]; ${ }^{2}$ Sheffield Teaching Hospitals NHS Foundation Trust, Sheffield, UK; ${ }^{3}$ Department of Pulmonology, University of Groningen, University Medical Center Groningen, Groningen, The Netherlands; ${ }^{4}$ Laboratory of Allergology \& Pulmonary Diseases, GRIAC Research Institute, University Medical Center Groningen, Groningen, The Netherlands; ${ }^{5}$ Laboratory of Allergology \& Pulmonary Diseases, University of Groningen, University Medical Center Groningen, Groningen, The Netherlands; ${ }^{6}$ Academic Unit of Immunology and Infectious Diseases, Department of Infection and Immunity, University of Sheffield, Sheffield, UK
}

Correspondence to Professor lan Sabroe, Academic Unit of Respiratory Medicine, Medical School, University of Sheffield, Beech Hill Road, Sheffield S10 2RX, UK; i.sabroe@sheffield.ac.uk their heart lie strong surface barriers, an effective mechanism of sensing pathogens through a range of pattern recognition receptors, ${ }^{2}$ the efficient killing of microorganisms by resident and recruited phagocytes, and efficient resolution of inflammation. ${ }^{3}$ Alveolar macrophages are the resident phagocytes, which along with epithelial cells and $\mathrm{T}$ cells coordinate an immune response that may require recruitment of other inflammatory cells such as neutrophils to clear invading pathogens. Clearance of bacteria such as pneumococci relies heavily on phagocyte competence which is optimised by $\mathrm{T}$ cell responses. When phagocyte capacity is stressed by intracellular pathogens, such as Mycobacterium species, there is a critical dependence on $\mathrm{T}$ cell function mediated via the IFN $\gamma / \mathrm{IL}-12 / \mathrm{IL}-23$ axis. ${ }^{4} 5$ Steroids, including ICS, have the potential to modify all such pathways.

Thus, we can happily trot out our favourite analogies describing the consequences of targeting the immune system ('doubleedged sword' is a particularly egregious example which every immunologist is required by some unwritten code to use intermittently). For now, we will stick with 'yin and yang', because any immunosuppression will modify the risks of infections while achieving therapeutic benefit. When considering the immunosuppression of ICS, a few messages have become clearer, but some have yet to be fully explored.

\section{MESSAGE 1: CORRECTLY USED, ICS SAVE LIVES}

This is not a surprise. Guideline-based management for asthma, focussing on the use of ICS in all but the mildest disease, has been the great life-saving and symptommodifying thrust of therapy over recent decades. The exact place of ICS and their optimal dosing remains more unclear in COPD, but TORCH clearly showed the potential for a fluticasone/salmeterol combination to reduce exacerbations. ${ }^{1}$ Nothing should detract from the correct therapeutic use. We have no good alternatives that can safely deliver local therapeutic manipulation of immune inflammation, and the benefits of ICS appear for now to clearly outweigh the risks of infectious complications.

\section{MESSAGE 2: ICS USE HAS SOME HITHERTO OVERLOOKED INFECTIVE RISKS}

So, do ICS actually increase the risk of some pulmonary infections? Recent studies suggest they may. TORCH linked ICS use with an increased risk of an epidemiological signature of community-acquired pneumonia, ${ }^{1}{ }^{6}$ data supported by other studies. ${ }^{7} \mathrm{~A}$ series of studies, including two recent articles in Thorax, have also linked ICS usage with increased risk of tuberculous or other mycobacterial infections. ${ }^{8-10}$ While these studies are epidemiological and not mechanistic, they encompass large populations, and their common message needs to give us pause for thought. Their complementary nature in differing populations reinforce the likely existence of mechanisms of mycobacterial control that are affected by ICS. Lee et al provide evidence that people from a population with a significant rate of $\mathrm{TB}$ latency show increased risk of active $\mathrm{TB}$ infection after treatment with steroids. ${ }^{10}$ Brassard et al show a similar risk of ICS-related TB infections, but in people less likely to have latent disease. ${ }^{9}$ Finally, Andrejak et al show a risk of ICS, particularly in the presence of structural lung disease, for non-tuberculous mycobacterial infection. ${ }^{8}$ Pneumococcal pneumonia and mycobacterial infections feature a prominent role for alveolar macrophages in pathogen killing and containment, and a requirement for $\mathrm{T}$ cell responses to optimise macrophage effector function. Mycobacteria reside in and replicate within macrophages, hidden from the worst the immune system can throw at them, living often in a state of relative suspended animation. Suppression of macrophage function, for example, with drugs targeting $\mathrm{TNF} \alpha$, increases risks of reactivation of $\mathrm{TB}$, and protocols are in place to define risk and screening for patients starting such therapies. Interestingly, among a myriad of effects of ICS, they reduce TNF $\alpha$ production by macrophages. ${ }^{11}$ ICS, such as fluticasone, can deplete T cells by induction of apoptosis, ${ }^{12}$ and in theory this could reduce the $\mathrm{T}$ cell responses required to aid macrophage control of specific pathogens.

Importantly, our genetic background heavily modifies the risks of developing the underlying lung disease, the efficacy of the epithelial barrier, and multiple aspects of the subsequent immune response to noxious stimuli and infections. Thus, whether the risks of ICS-mediated immunosuppression are actually very small in some individuals, and much greater than 
we are aware of in others, is not yet known, but may well be the case. Furthermore, differences may exist between ICS molecules, since Andréjak et al noted greater odds of non-tuberculous mycobacterial infection in patients on fluticasone versus budesonide (BUD) ${ }^{8}$ and we will return to this theme below.

These new data remind us again of the necessity to use ICS at the lowest appropriate dose to maintain good control of detrimental inflammation within established guideline-based management, and to be cautious of assumptions extending their use beyond where there is a good evidence base.

\section{MESSAGE 3: ICS MIGHT PROTECT AGAINST SOME INFECTIONS?}

The majority of asthma exacerbations, and many of COPD, are viral in nature. ${ }^{13}$ Asthmatics have a pre-existing immune deficit such that their epithelial cells fail to produce some interferons in response to viral infections. ${ }^{14}$ Yet despite the determinedly suppressive actions of ICS on viral-induced proinflammatory cytokine production by the epithelium, in some circumstances ICS appear to be the opposite of immunosuppressive: infective exacerbations of COPD are lessened by appropriate use of ICS in the right patients, and asthmatics with sufficient ICS to control their disease experience fewer clinically significant respiratory infections.

The mechanisms underlying steroidinduced resistance to infection, or the sequelae of infection, are less clearly defined. ICS use will decrease excessive inflammatory responses to infections, limiting lung damage. Additionally, reductions in persistent inflammation may restore a more normal epithelial and airway architecture, which will enable a response to infection that is more typical of the healthy airway. In keeping with this idea, we have recently observed that ICS counteract the airway epithelial barrier dysfunction induced by a viral mimic in vitro, ${ }^{15}$ which may lead to reduced accessibility of pathogens to submucosal inflammatory cells in vivo. Hinting again at potentially relevant differences between ICS molecules, BUD protects better against cigarette smoke-induced epithelial barrier dysfunction than fluticasone propionate (FP), ${ }^{15}$ while fluticasone furoate is more effective than FP or BUD in protecting epithelial cells from elastase-induced damage. ${ }^{16}$ BUD-based regimes may also be associated with fewer pneumonia events in people with COPD than FP-based treatments. ${ }^{17}$
Animal models show that viral airways infections can cause a profound persistent impairment of innate immune responses to bacteria, increasing risks of secondary bacterial infections. ${ }^{18}$ Furthermore, while ICS dampen production of proinflammatory cytokines, they might also stimulate an increase in the pathogen-driven expression of other epithelial-derived host defence molecules. ${ }^{19}$ Thus, ICS treatment may limit an excessive response to viral pathogens, and thus help reduce persistent immune tolerance in the airway, and through increases in or preserved production of antimicrobial molecules ${ }^{19}$ reduce the risks or severity of secondary bacterial infections.

\section{CONCLUSIONS}

ICS are profoundly beneficial in the right circumstances, when used in guidelinebased and evidence-based strategies. It is becoming evident that people treated with ICS are at increased risk of mycobacterial and other bacterial infections, and this should be borne in mind when monitoring people on these drugs, but ICS also have protective roles, reducing risks and harm of some infections. Future genetic studies combined with epidemiological approaches may define those at greatest risk of specific infectious complications of ICS use. We also need clearer data determining if risks are particularly associated with individual ICS molecules, and if so, whether this simply results from their relative potency, perhaps modulated by genetic factors in the treated individual, or through other subtleties of their mechanisms. It is also likely that a better understanding of these risks will provide new insights into the pathogenesis of pulmonary infection. We can hope that non-ICS anti-inflammatory drugs will come forward and change the landscape of disease, but like almost all diseasemodifying anti-inflammatory drugs, these will almost certainly also be associated with generic or therapy-specific infective risks, as part of the inevitable yin and yang of targeting a set of pathways designed through evolution to stop us dying from or being significantly injured by pathogens.

Acknowledgements The authors are grateful to Prof Stuart Farrow (GSK and Manchester University) for critical reading of the manuscript.

Contributors This editorial was written by IS, DP, IH and DD in partnership.

Funding None.

Competing interests None.
Provenance and peer review Commissioned; internally peer reviewed.

To cite Sabroe I, Postma D, Heijink I, et al. Thorax 2013:68:1085-1087.

Published Online First 8 August 2013

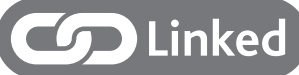

http://dx.doi.org/10.1136/thoraxjnl-2012-203175

Thorax 2013:68:1085-1087.

doi:10.1136/thoraxjnl-2013-203773

\section{REFERENCES}

1 Calverley PM, Anderson JA, Celli B, et al. Salmeterol and fluticasone propionate and survival in chronic obstructive pulmonary disease. $N$ Engl J Med 2007;356:775-89.

2 Chaudhuri N, Whyte MKB, Sabroe I. Reducing the toll of inflammatory lung disease. Chest 2007:131:1550-6.

3 Dockrell DH, Whyte MKB, Mitchell TJ. Pneumococcal pneumonia: mechanisms of infection and resolution. Chest 2012;142:482-91.

4 Fieschi C, Dupuis S, Catherinot E, et al. Low penetrance, broad resistance, and favorable outcome of interleukin 12 receptor beta1 deficiency: medical and immunological implications. J Exp Med 2003; 197:527-35.

5 Marriott HM, Daigneault M, Thompson AAR, et al. A decoy receptor 3 analogue reduces localised defects in phagocyte function in pneumococcal pneumonia. Thorax 2012:67:985-92.

6 Crim C, Calverley PMA, Anderson JA, et al. Pneumonia risk in COPD patients receiving inhaled corticosteroids alone or in combination: TORCH study results. Eur Respir J 2009:34:641-7.

7 Ernst P, Gonzalez AV, Brassard P, et al. Inhaled corticosteroid use in chronic obstructive pulmonary disease and the risk of hospitalization for pneumonia. Am J Respir Crit Care Med 2007;176:162-6.

8 Andréjak C, Nielsen R, Thomsen VØ, et al. Chronic respiratory disease, inhaled corticosteroids and risk of non-tuberculous mycobacteriosis. Thorax 2013:68:256-62.

9 Brassard P, Suissa S, Kezouh A, et al. Inhaled corticosteroids and risk of tuberculosis in patients with respiratory diseases. Am J Respir Crit Care Med 2011;183:675-8.

10 Lee $\mathrm{CH}$, Kim K, Hyun MK, et al. Use of inhaled corticosteroids and the risk of tuberculosis. Thorax 2013:68:1105-13.

11 Marshall BG, Wangoo A, Harrison LI, et al. Tumour necrosis factor-alpha production in human alveolar macrophages: modulation by inhaled corticosteroid Eur Respir J 2000;15:764-70.

12 O'Sullivan S, Cormincan L, Burke CM, et al. Fluticasone induces $\mathrm{T}$ cell apoptosis in the bronchial wall of mild to moderate asthmatics. Thorax 2004;59:657-61.

13 Johnston S. Overview of virus-induced airway disease. Proc Am Thorac Soc 2005:2:150-6.

14 Wark PA, Johnston SL, Bucchieri F, et al. Asthmatic bronchial epithelial cells have a deficient innate immune response to infection with rhinovirus. J Exp Med 2005;201:937-47.

15 Heijink $\mathrm{IH}$, van den Berge $\mathrm{M}$, Jonker $\mathrm{MR}$, et al. Comparing the effects of fluticasone propionate and 
budesonide on airway epithelial barrier function. $A m$ J Respir Crit Care Med 2012;185:A6796.

16 Salter M, Biggadike K, Matthews JL, et al.

Pharmacological properties of the enhanced-affinity glucocorticoid fluticasone furoate in vitro and in an

in vivo model of respiratory inflammatory disease.

Am J Physiol-Lung C 2007;293:L660-7.
17 Halpin DMG, Gray J, Edwards SJ, et al. Budesonide/ formoterol vs. salmeterol/fluticasone in COPD: a systematic review and adjusted indirect comparison of pneumonia in randomised controlled trials. Int $J$ Clin Pract 2011;65:764-74.

18 Didierlaurent A, Goulding J, Patel S, et al. Sustained desensitization to bacterial Toll-like receptor ligands after resolution of respiratory influenza infection. J Exp Med 2008;205:323-9.

19 Zhang N, Truong-Tran QA, Tancowny B, et al. Glucocorticoids enhance or spare innate immunity: effects in airway epithelium are mediated by CCAAT/ enhancer binding proteins. I Immunol 2007;179:578-89 